\title{
Comparison of Patient Survival after Resection for Pulmonary Carcinoid Tumors Compared to Other Neuroendocrine Tumors: A United States Population Study
}

\author{
Jay M. Bhatt, J. Nilas Young, David T. Cooke* \\ Division of Cardiothoracic Surgery, Davis Medical Center, University of California, Sacramento, USA. \\ Email: *david.cooke@ucdavis.ucdmc.edu
}

Received October $9^{\text {th }}, 2012$; revised November $9^{\text {th }}, 2012$; accepted November $19^{\text {th }}, 2012$

\begin{abstract}
Background: We hypothesized: 1) Resected pulmonary typical carcinoid (TC) will show a favorable clinical pattern compared to other bronchopulmonary neuroendocrine tumors (BPNETs); and 2) Atypical carcinoid (AC) and large-cell neuroendocrine carcinoma (LCNEC) patients will have similar outcomes. Methods: The Surveillance Epidemiology and End Result database was queried to compare demographics and tumor specific variables in patients undergoing resection for TC, AC, LCNEC and small cell lung cancer (SCLC) from 2001 to 2006. Results: Similar to LCNEC, AC patients had greater incidence of histologic positive lymph nodes compared to TC $(\mathrm{P}<0.001)$. Survival analysis showed a mean survival of $40.0 \pm 1.9$ months for SCLC, $46.2 \pm 2.2$ months for LCNEC, $58.3 \pm 2.3$ months for AC, and 70.2 \pm 0.2 months for TC tumors. TC patients demonstrated favorable survival, and SCLC patients poorer survival, compared to AC and LNEC patients $(\mathrm{P}<0.01)$. Conclusion: AC tumors are a BPNET histology that exhibits clinical behavior distinct from TC. AC tumors should be staged, and treated with stage appropriate therapeutic strategies similar to other non-small cell lung cancers.
\end{abstract}

Keywords: Lung Cancer; Carcinoid; Neuroendocrine Tumor; Thoracic Surgery

\section{Introduction}

In the 1999 World Health Organization classification atypical carcinoid (AC) and typical carcinoid (TC) tumors are considered "carcinoid type". Carcinoid type is a sub-category of the bronchopulmonary neuroendocrine tumor (BPNET) classification that includes large-cell neuroendocrine carcinoma (LCNEC), and small cell lung cancer (SCLC) [1].

$\mathrm{AC}$ tumors have neuroendocrine features of being organoid, trabecular, palisading, and rosette-like, with 2 to 10 mitotic figures seen in a 10 high powered focus [2]. Although AC tumors are distinguished from $\mathrm{TC}$ on histological criteria such as $>2$ mitotic figures per high power field, both show common histological features of spindle, oncocytic and melanocytic cells.

The above four types of BPNET represent nearly $20 \%$ of tumors found in the lung. AC and TC tumors comprise of about $1 \%$ of the BPNETs with an incidence rate of about 1.57 in 100,000 as of 2003 , representing an increase of $6 \%$ per year over the last 30 years [3]. Iyoda

${ }^{*}$ Corresponding author. et al. demonstrated that the $\mathrm{AC}$ and $\mathrm{TC}$ tumors are not related to smoking, and are found in younger patients [4]. Small studies suggest that AC caries a worse prognosis than TC [5-8]. In this study, using a large population cancer database, we tested the hypotheses 1) AC caries a worse prognosis than TC; 2) TC, AC, LNEC and SCLC represent a stepwise increasing negative prognosis within the BPNET classification; and 3) patient outcomes for $\mathrm{AC}$ tumors are similar to those for LNEC tumors.

\section{Methods}

\subsection{Database}

The Surveillance Epidemiology and End Result (SEER) database was queried to compare AC to TC, LCNEC and SCLC. The SEER Program is a National Cancer Institute source that provides information regarding incidence and survival of various cancers in the United States. The database represents roughly $26 \%$ of US population, including $23 \%$ of African Americans, $40 \%$ of all Hispanics, $42 \%$ of American Indians/Alaskan natives, $53 \%$ of 
Asians, and $70 \%$ of Hawaiian/Pacific Islanders [9].

Data obtained from SEER was used to determine demographic variables such as age, gender, and ethnicity. In addition, tumor specific variables such as AJCC pathologic stage, laterality, primary site, nodal status and tumor grade were evaluated. The University of California, Davis Medical Center Institutional Review Board determined that this project qualified for an exemption.

\subsection{Search Criteria}

Inclusion criteria included all post-lobectomy or similar procedures performed for these four histologic types from 2001-2006. 2001 was selected as the start of the analysis as it was the first year available in the database for information on AC tumors. Other surgical procedures considered included segmental resection, bronchial sleeve resection, lobectomy with mediastinal node dissection, lobe or bilobectomy extended, with or without chest wall/pericardium/diaphragm, pneumonectomy of any type with or without mediastinal node dissection, extended pneumonectomy, resection of the lung NOS, or surgery NOS.

Subsequently, patients with single primary at initial diagnosis, along with only the first occurrence of malignancy were evaluated. Disease specific survival endpoint was death from "Lung and Bronchus" as coded in the SEER database. The search criteria identified 2239 patients undergoing lobectomy for TC, 145 for AC, 510 for LCNEC and 1521 for SCLC from 2001 to 2006.

\subsection{Statistical Analysis}

The significance of mean age calculation was determined using analysis of variance (ANOVA). Categorical outcomes such as number of primaries, AJCC Stage, histologic type, primary site, nodal status, sex, race and grade were compared using the Pearson Chi-Square test.

The data obtained from the search was used to construct a Kaplan-Meier survival curve to determine survival of the patients following surgical resection. Survival analysis was performed for each histologic subtype. A multivariate Cox regression analysis was also performed to evaluate the importance of these variables in the survival patterns of each of the four histologic types.

Analysis was performed with PASW Statistics version 17.0 (SPSS Inc, Chicago, IL). Statistical significance was defined as a P-value of 0.05 or less.

\section{Results}

\subsection{Demographics}

Atypical carcinoid tumors occur in patients at a younger age (mean 59.9 years of age \pm 13.5 ) than LNEC and
SCLC $(65.3 \pm 11.0$ and $66.2 \pm 10.1$ respectively $)$ but older than patients with typical carcinoid tumors $(58.5 \pm$ 15.7, P < 0.001; Table 1). In regards to gender, AC, TC and SCLC occurred at a higher rate in females compared to males $(70.3 \%, 68.7 \%$ and $51.4 \%$ respectively, $\mathrm{P}<$ 0.001 ) while LCNEC was more prevalent in males (Figure 1). There was no difference $(\mathrm{P}=0.29)$ between groups in regards to ethnicity (data not shown).

\subsection{Tumor Characteristics}

Tumor specific variables including anatomic location, stage and lymph node involvement are illustrated in Table 1. The majority of AC, LNEC and SCLC tumors occurred in the upper lung fields (i.e. upper, middle and lingula; $53.1 \%, 65.3,64.1 \%$ and $49.6 \%$ ). TC had a higher predilection to lower lobe incidence $(41 \%, \mathrm{P}<0.001)$. All histologies had a higher predilection towards the right lung $(\mathrm{P}<0.001)$.

The majority of tumor grades for AC, TC and SCLC were characterized in the SEER database as "Unknown". Of known data values for tumor grade, the majority of TC and AC tumors were Grade I/II $(93.8 \%$ and $87.8 \%$ respectively, $\mathrm{P}<0.001$; Table 1) and LNEC and SCLC were mostly Grade III/IV (94.3\% and $95.8 \%$ respectively, $\mathrm{P}<0.001)$. AC had a similar percentage of patients with positive lymph nodes as LCNEC (30.4\%) and SCLC (46.3\%). Although very few TC patients had positive lymph nodes compared to the other histologies $(\mathrm{P}<$ $0.001), 8.7 \%$ of patients with TC presented with positive lymph nodes. In regards to tumor stage, AC, TC and LCNEC tumors were mostly Stage Occult/I/II, and SCLC mostly Stage III/IV (P $<0.001$; Table 2).

\subsection{Survival}

Survival analysis for all stages showed a mean survival

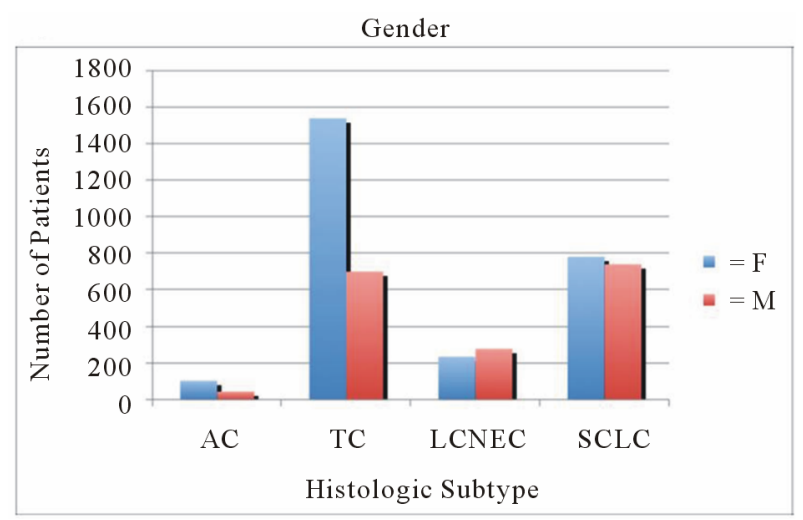

Figure 1. Predilection of TC and AC incidence towards female patients. AC = atypical carcinoid, $\mathrm{TC}=$ typical carcinoid, LCNEC = large cell neuroendocrine carcinoma, SCLC $=$ small cell lung cancer, $\mathbf{F}=$ Female, $M=$ Male. 
Table 1. Patient clinical and tumor characteristics.

\begin{tabular}{|c|c|c|c|c|c|c|c|c|c|c|c|c|c|}
\hline & & $\mathrm{AC}$ & & & $\mathrm{TC}$ & & & LCNEC & & & SCLC & & \\
\hline Characteristic & $\mathrm{N}$ & Mean & $\mathrm{SD}$ & $\mathrm{N}$ & Mean & $\mathrm{SD}$ & $\mathrm{N}$ & Mean & $\mathrm{SD}$ & $\mathrm{N}$ & Mean & $\mathrm{SD}$ & P-value \\
\hline \multirow[t]{2}{*}{ Age } & 145 & 59.9 & 13.5 & 2239 & 58.5 & 15.7 & 510 & 65.3 & 11.0 & 1521 & 66.2 & 10.1 & $<0.001$ \\
\hline & Count & Percent & & Count & Percent & & Count & Percent & & Count & Percent & & P-value \\
\hline $\begin{array}{l}\text { Patients with } \\
\text { Positive Nodes }\end{array}$ & 39 & 26.9 & & 194 & 8.7 & & 155 & 30.4 & & 704 & 46.3 & & $<0.001$ \\
\hline Laterality & & & & & & & & & & & & & $<0.001$ \\
\hline Left & 55 & 38.0 & & 846 & 38.0 & & 225 & 44.0 & & 614 & 42.0 & & \\
\hline Right & 90 & 62.0 & & 1376 & 61.0 & & 281 & 55.0 & & 802 & 53.0 & & \\
\hline Tumor Lobe & & & & & & & & & & & & & $<0.001$ \\
\hline $\begin{array}{l}\text { Upper, Lingula, } \\
\text { Middle }\end{array}$ & 77 & 53.1 & & 1057 & 47.2 & & 327 & 64.1 & & 755 & 49.6 & & \\
\hline Lower & 53 & 36.6 & & 918 & 41.0 & & 145 & 28.4 & & 292 & 19.2 & & \\
\hline Grade & & & & & & & & & & & & & $<0.001$ \\
\hline Grade I \& II & 43 & $87.8^{*}$ & & 180 & $93.8^{*}$ & & 18 & $5.7^{*}$ & & 29 & $4.2^{*}$ & & \\
\hline Grade III \& IV & 6 & $12.2^{*}$ & & 12 & $6.2^{*}$ & & 298 & $94.3^{*}$ & & 667 & $95.8^{*}$ & & \\
\hline Unknown & 96 & & & 1791 & & & 189 & & & 801 & & & \\
\hline
\end{tabular}

"Percent of patients with tumors with known grade; $\mathrm{AC}=$ atypical carcinoid; TC = typical carcinoid; $\mathrm{LCNEC}=$ large cell neuroendocrine carcinoma; $\mathrm{SCLC}=$ small cell lung cancer.

Table 2. Tumor stage characteristics.

\begin{tabular}{|c|c|c|c|c|c|c|c|c|c|}
\hline \multirow{2}{*}{ Stage ${ }^{*}$} & \multicolumn{2}{|c|}{$\mathrm{AC}$} & \multicolumn{2}{|c|}{$\mathrm{TC}$} & \multicolumn{2}{|c|}{ LCNEC } & \multicolumn{2}{|c|}{ SCLC } & \multirow[b]{2}{*}{ P-value } \\
\hline & Count & Percent ${ }^{* *}$ & Count & Percent ${ }^{* *}$ & Count & Percent ${ }^{* *}$ & Count & Percent ${ }^{* *}$ & \\
\hline Occult I, IA, IB & 31 & 63.2 & 147 & 76.6 & 176 & 55.7 & 205 & 29.5 & $<0.001$ \\
\hline IIA, IIB & 3 & 6.1 & 11 & 5.7 & 44 & 13.9 & 65 & 9.3 & \\
\hline IIIA & 4 & 8.2 & 11 & 5.7 & 47 & 14.9 & 111 & 15.9 & \\
\hline IIIB & 7 & 14.3 & 13 & 6.8 & 22 & 7.0 & 114 & 16.4 & \\
\hline IV & 4 & 8.2 & 10 & 5.2 & 27 & 8.5 & 201 & 28.9 & \\
\hline Unknown & 4 & & 1205 & & 12 & & 74 & & \\
\hline
\end{tabular}

*6th Edition, AJCC Stage; ${ }^{* *}$ Percent of patients with tumors of known grade; AC = atypical carcinoid; TC = typical carcinoid; LCNEC = large cell neuroendocrine carcinoma; $\mathrm{SCLC}=$ small cell lung cancer.

of $40.0 \pm 1.9$ months for SCLC (95\% CI $36.4-43.7$ months), $46.2 \pm 2.2$ months for LCNEC (95\% CI 42.0 50.4 months), $58.3 \pm 2.3$ months for AC (95\% CI 53.9 62.7 months), and $70.2 \pm 0.2$ months for TC tumors (95\% CI 69.8 - 70.6 months). TC patients demonstrated the most favorable survival and SCLC patients the poorest survival, with a Mantel-Cox log rank P-value of $<0.01$ between the four groups). Five-year survivals for the histologies were $98 \%$ for TC patients, $82 \%$ for AC patients, $50 \%$ for LCNEC and $42 \%$ for SCLC patients (Figure 2).

Multivariate Cox analysis revealed that increasing age at diagnosis $(\mathrm{P}=0.003)$ and increasing stage $(\mathrm{P} \leq 0.001)$ were negative predictors of survival, with stage III and IV tumors having the least probability of surviving (Table 3). 


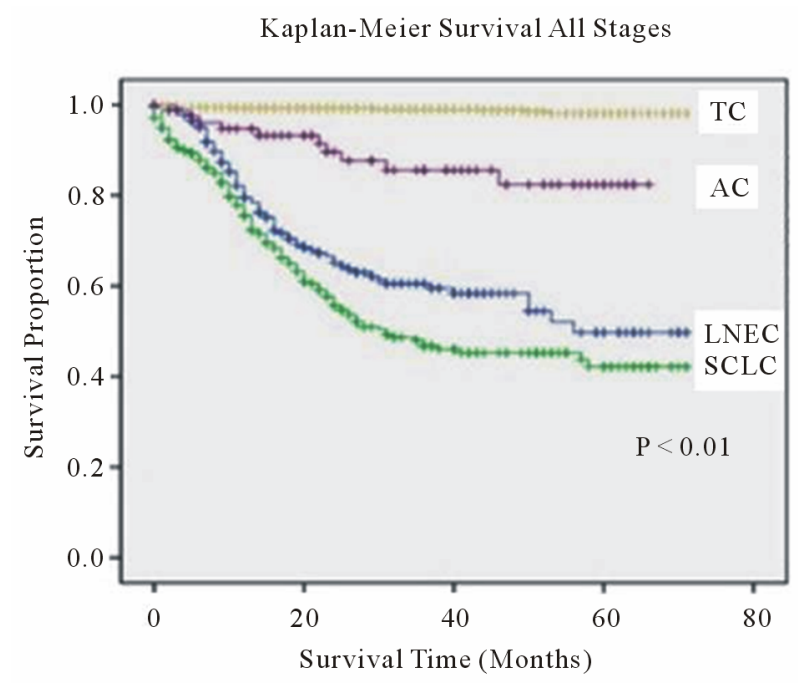

Figure 2. Kaplan-Meier 5-year survival analysis for all stages, post-lobectomy. Reveals a mean survival of $40.0 \pm$ 1.9 months for SCLC, $46.2 \pm 2.2$ months for LCNEC, $58.3 \pm$ 2.3 months for AC and $70.2 \pm$ months for TC tumors. Mantel-Cox log rank P-value of $<0.01$ between the four groups. AC $=$ atypical carcinoid, $\mathbf{T C}=$ typical carcinoid, LCNEC = large cell neuroendocrine carcinoma, SCLC = small cell lung cancer.

Table 3. Multivariate cox analysis.

\begin{tabular}{|c|c|c|c|c|}
\hline \multirow{2}{*}{ Variable } & \multirow{2}{*}{ P-value } & \multirow{2}{*}{$\begin{array}{l}\text { Odds } \\
\text { Ratio }\end{array}$} & \multicolumn{2}{|c|}{$95.0 \%$ CI for Odds Ratio } \\
\hline & & & Lower & Upper \\
\hline \multicolumn{5}{|l|}{ Stage $^{*}$} \\
\hline Stage 1 & $<0.001$ & 4.63 & 2.31 & 9.26 \\
\hline Stage 2 & 0.011 & 2.63 & 1.25 & 5.56 \\
\hline Stage 3 & 0.067 & 2.03 & 0.95 & 4.30 \\
\hline Stage 4 & 0.037 & 2.33 & 1.05 & 5.18 \\
\hline Age & 0.003 & 0.97 & 0.96 & 0.99 \\
\hline
\end{tabular}

*6th Edition, AJCC Stage.

\section{Discussion}

This study is a large retrospective analysis that seeks to differentiate clinical outcomes of resected atypical pulmonary carcinoid tumors compared to typical carcinoid tumors. In addition, as a point of reference, we compared post resection $\mathrm{AC}$ and TC outcomes to NSLC and SCLC that are part of the same BPNET class. AC tumors demonstrated distinct tumor specific variables than TC tumors. SCLC had the highest number positive lymph nodes discovered with the tumor, followed by LCNEC, then AC, and TC, which had the lowest. This correlates directly with the observed post surgical survival pattern, and these results confirm that patients with $\mathrm{AC}$ tumors had significantly worse post lobectomy survival compared to patients with TC tumors. In addition, AC patients had similar percentage lymph node positivity to LNEC (28.9\% to $30.4 \%)$.

Our analysis expands on and confirms previously published smaller single institutional studies that evaluated pulmonary atypical carcinoid tumors and their survival patterns. Cao et al found that patients with $\mathrm{AC}$ presented with higher stage disease [5]. Garcia-Yuste et al. found a $78 \% 5$-year survival for patients with $\mathrm{AC}$ tumors with no lymph node involvement, and $60 \%$ survival with nodal involvement. Survival for patients with $\mathrm{TC}$ was significantly better than $\mathrm{AC}$ counterparts [6]. Bini et al. found the best overall survival for patients with AC tumors (88\%), which was marginally worse than patients with TC [7]. In a large European cancer registry comparison (265 total carcinoid tumors), 5-year survival for resected TC was $96 \%$ compared to $79 \%$ for AC [8]. Zhong et al. in an analysis of 131 consecutive patients undergoing pulmonary resection also found a survival advantage for TC tumors [10].

It is interesting to note that in our analysis, $8.7 \%$ of TC tumors had lymph node involvement. This may be a result of misclassification of AC as TC. However, if this misclassification was very prevalent, then our results should confirm the null hypothesis, or no survival advantage for $\mathrm{TC}$, and that is not the case. The observation of $8.7 \%$ of TC tumors with nodal involvement underscores the important of standard surgical therapy for TC tumors in patients with appropriate performance status, including pulmonary resection with mediastinal lymph node sampling or dissection. This is important as a recent study demonstrated that only $57.3 \%$ of patients had mediastinal lymph nodes removed at the time of pulmonary resection for NSCLC [11]. Also there have been reports advocating local, endobronchial therapy alone for carcinoid tumors [12]. Patients undergoing only an endobronchial treatment algorithm may be understaged.

In our analysis, patients with SCLC exhibited an unusually high 5 -year survival $(>40 \%)$. The patient populations examined are patients who have undergone pulmonary resection, are therefore a highly selected group. Although it is impossible to determine, as the data is de-identified, most of the SCLC tumors may have been confirmed histologically for patients undergoing resection for suspicious solitary pulmonary nodules, without pre-operative needle biopsy, given surgery is rarely the upfront therapy of choice for SCLC.

Limitations of our study include those inherent to analysis of a large national database. The SEER Database only represents approximately $26 \%$ of the US population diagnosed with cancer. In addition, there is a significant 
racial disparity among reporting of minority patients. For example, only $23 \%$ of African Americans are represented in the database, whereas other minorities have up to $70 \%$ representation in the database [9]. The study was performed using the AJCC 6th edition, which is the edition utilized by the SEER database for the study period. Travis et al. also examined the SEER database including 1437 surgical broncho-pulmonary carcinoid cases, and came to the conclusion that the 7 th edition of the TNM staging system be applied to these histologic cohorts [13].

\section{Conclusion}

Based on our results, we conclude that AC tumors should be staged, and treated with stage appropriate neoadjuvant and adjuvant therapies similar to other NSCLCs. This approach may provide patients with maximum benefits of therapy and the best chance for survival. Finding the appropriate chemotherapeutic regimen for carcinoid tumors may be challenging.

\section{Acknowledgements}

This work was supported in part by the 2009 American Association for Thoracic Surgery Summer Intern Scholarship.

\section{REFERENCES}

[1] National Cancer Institute, "Non-Small Cell Lung Cancer Treatment," 2010. http://www.cancer.gov/cancertopics/pdq/treatment/non-s mall-cell-lung/HealthProfessional/page3

[2] C. A. Moran, S. Suster, Coppola and M. R. Wick, "Neuroendocrine Carcinomas of the Lung: A Critical Analysis," American Journal of Clinical Pathology, Vol. 131, No. 2, 2009, pp. 206-221. doi:10.1309/AJCP9H1OTMUCSKQW

[3] B. I. Gustafsson, M. Kidd, A. Chan, M. V. Malfertheiner and I. M. Modlin, "Bronchopulmonary Neuroendocrine Tumors," Cancer, Vol. 113, No. 1, 2008, pp. 5-21. doi:10.1002/cncr.23542

[4] A. Iyoda, K. Hiroshima, M. Baba, Y. Saitoh, H. Ohwada and T. Fujisawa, "Pulmonary Large Cell Carcinomas with Neuroendocrine Features Are High-Grade Neuroendocrine Tumors," The Annals of Thoracic Surgery, Vol. 73, No. 4, 2002, pp. 1049-1054. doi:10.1016/S0003-4975(01)03616-5
[5] C. Cao, T. D. Yan, C. Kennedy, N. Hendel, P. G. Bannon and B. C. McCaughan, "Bronchopulmonary Carcinoid Tumors: Long-Term Outcomes after Resection," Annals of Thoracic Surgery, Vol. 91, No. 2, 2011, pp. 339-343. doi:10.1016/j.athoracsur.2010.08.062

[6] M. García-Yuste, J. M. Matilla, A. Cueto, J. M. Paniagua, G. Ramos, M. A. Cañizares and I. Muguruza, "Typical and Atypical Carcinoid Tumours: Analysis of the Experience of the Spanish Multi-Centric Study of Neuroendocrine Tumours of the Lung," European Journal of Cardiothoracic Surgery, Vol. 31, No. 2, 2007, pp. 192-197. doi:10.1016/j.ejcts.2006.11.031

[7] A. Bini, J. Brandolini, N. Cassanelli, F. Davoli, G. Dolci, F. Sellitri and F. Stella, "Typical and Atypical Pulmonary Carcinoids: Our Institutional Experience," Interactive Cardiovascular and Thoracic Surgery, Vol. 7, No. 3, 2008, pp. 415-418. doi:10.1510/icvts.2007.173328

[8] A. Naalsund, H. Rostad, E. H. Strøm, M. B. Lund and T. E. Strand, "Carcinoid Lung Tumors-Incidence, Treatment and Outcomes: A Population-Based Study," European Journal of Cardiothoracic Surgery, Vol. 39, No. 4, 2011, pp. 565-569. doi:10.1016/j.ejcts.2010.08.036

[9] National Cancer Institute, U.S. National Institutes of Health, "Overview of SEER Program," 2010. http://seer.cancer.gov/about/

[10] C. X. Zhong, F. Yao, H. Zhao, J. X. Shi and L. M. Fan, "Long-Term Outcomes of Surgical Treatment of Pulmonary Carcinoid Tumors: 20 Years' Experience with 131 Patients," Chinese Medical Journal (English), Vol. 125, No. 17, 2012, pp. 3022-3026.

[11] A. G. Little, V. W. Rusch, J. A. Bonner, L. E. Gaspar, M. R. Green, W. R. Webb and A. K. Stewart, "Patterns of Surgical Care of Lung Cancer Patients," The Annals of Thoracic Surgery, Vol. 80, No. 6, 2005, pp. 2051-2056. doi:10.1016/j.athoracsur.2005.06.071

[12] L. Bertoletti, R. Elleuch, D. Kaczmarek, R. Jean-François and J. M. Vergnon, "Bronchoscopic Cryotherapy Treatment of Isolated Endoluminal Typical Carcinoid Tumor," Chest, Vol. 130, No. 5, 2006, pp. 1405-1411. doi:10.1378/chest.130.5.1405

[13] W. D. Travis, D. J. Giroux, K. Chansky, H. Asamura, E. Brambilla, J. Jett, C. Kennedy, R. Rami-Porta, V. W. Rusch and P. Goldstraw, "The IASLC Lung Cancer Staging Project: Proposals for the Inclusion of Broncho-Pulmonary Carcinoid Tumors in the Forthcoming (Seventh) Edition of the TNM Classification for Lung Cancer," Journal of Thoracic Oncology, Vol. 3, No. 11, 2008, pp. 1213-1223. doi:10.1097/JTO.0b013e31818b06e3 